\title{
A Relic of a Bygone Age? \\ Causation, Time Symmetry and the Directionality Argument
}

\author{
Matt Farr \& Alexander Reutlinger ${ }^{\dagger}$
}

February 15, 2013

\begin{abstract}
Bertrand Russell famously argued that causation is not part of the fundamental physical description of the world, describing the notion of cause as "a relic of a bygone age" (Russell, 1913, p. 1). This paper assesses one of Russell's arguments for this conclusion: the 'Directionality Argument', which holds that the time symmetry of fundamental physics is inconsistent with the time asymmetry of causation. We claim that the coherence and success of the Directionality Argument crucially depends on the proper interpretation of the 'time symmetry' of fundamental physics as it appears in the argument, and offer two alternative interpretations. We argue that: (1) if 'time symmetry' is understood as the time-reversal invariance of physical theories, then the crucial premise of the Directionality Argument should be rejected; and (2) if 'time symmetry' is understood as the temporally bidirectional nomic dependence relations of physical laws, then the crucial premise of the Directionality Argument is far more plausible. We defend the second reading as continuous with Russell's writings, and consider the consequences of the bidirectionality of nomic dependence relations in physics for the metaphysics of causation.
\end{abstract}

\section{Introduction}

In his paper "On the Notion of Cause," Bertrand Russell famously argues that-contrary to the beliefs of philosophers-causation is not among the building blocks of the world as

\footnotetext{
${ }^{*}$ Forthcoming in Erkenntnis.

$\dagger^{\top}$ matt.farr@sydney.edu.au / http://sites.google.com/site/mwefarr/

$\bowtie$ alexander.reutlinger@uni-koeln.de/http://uni-koeln.academia.edu/alexanderreutlinger/ This paper is fully co-authored. The authors' names appear in alphabetical order only.
} 
described by fundamental physics. Russell held that notion of cause is a "relic of a bygone age" of physics, which no longer plays a role in fundamental physics (Russell, 1913, p. 1). Many philosophers of physics today support this claim that causal relations do not belong to the ontology suggested by fundamental physics. ${ }^{1}$ One important argument for this claim is the Directionality Argument, the success of which depends on characterising the dynamical laws of fundamental physics as being time-symmetric. In this paper, we argue that: (1) time symmetry is an ambiguous concept as used in the debate; and (2) only one of two possible disambiguations of 'time symmetry' provides a plausible version of the Directionality Argument.

Russell (1913) presents a number of arguments for the conclusion that there is no place for causation in fundamental physics. We focus on one particular argument: the Directionality Argument. In order to properly understand this argument, it is important to acknowledge that Russell ascribes various characteristic features to causal relations:

a. Sufficiency. Causes are sufficient for their effects; it always is the case that if the cause occurs, then the effect occurs (Russell, 1913, pp. 7-12). Causes "compel" (Russell, 1913, p. 2, pp. 9-10), enforce, or produce their effects, i.e. some kind of modal connection obtains among cause and effect.

b. Locality. Cause $c$ and effect $e$ are local and distinct events in a spacetime region $r$ where $r$ is "something short of the whole state of the universe" (Russell, 1913, p. 7).

c. Causal Asymmetry. The causal relation is asymmetric: if $A$ causes $B$, then $B$ does not cause $A$ (Russell, 1913, p. 10).

d. Causal Time Asymmetry. Causes precede their effects in time, but not vice versa (Russell, 1913, pp. 13-16).

In the current literature on the metaphysics of causation, these features of causal relations are thought of as explicating the folk notion of causation (Norton, 2007, pp. 36-38; Ladyman and Ross, 2007, p. 268; Ross and Spurrett, 2007, p. 13). As these philosophers point out, causation is often characterised by these features not only in ordinary discourse but also in special science discourse. In this respect it is misleading to call the notion of

\footnotetext{
${ }^{1}$ In the literature, some philosophers also endorse the stronger claim that if an entity is physically fundamental, then it is metaphysically fundamental. We do not require the truth of this stronger claim for the rest of the paper, as we are exclusively interested in whether causation is part of the metaphysics of fundamental physics. However, our positive proposal in Section 3 is consistent with the truth of the stronger claim.
} 
causation a 'folk' notion. However, we here adopt the terms 'folk notion' and 'folk features' of causation, since they are established and useful terms in the debate. Presupposing that these features (a)-(d) characterise causal relations, Russell argues that the relations of physics (especially nomic relations) lack precisely these features, and, thus, relations in physics are not causal. Note that we ignore the features of sufficiency and locality in this paper and focus on the features of causal asymmetry and causal time asymmetry.

Concerning the features of time asymmetry and asymmetry of causation, Russell points out a fact about fundamental dynamical equations in physics that he illustrates using the example of the law of gravitation:

In the motions of mutually gravitating bodies, there is nothing that can be called a cause, and nothing that can be called an effect; there is merely a formula. Certain differential equations can be found, which hold at every instant for every particle of the system, and which, given the configuration and velocities at one instant, or the configurations at two instants, render the configuration at any other earlier or later instant theoretically calculable. That is to say, the configuration at any instant is a function of that instant and the configurations at two given instants. This statement holds throughout physics, and not only in the special case of gravitation. But there is nothing that could be properly called "cause" and nothing that could be properly called "effect" in such a system. (Russell, 1913, p. 14; emphasis added.)

The law [of gravitation] makes no difference between past and future: the future 'determines' the past in exactly the same sense in which the past 'determines' the future. The word 'determine', here, has a purely logical significance: a certain number of variables 'determine' another variable if that variable is a function of them. (Russell, 1913, p. 15; emphasis added.)

Note that the first of these two passages primarily concerns the asymmetry of causation, whereas the latter concerns the time asymmetry of causation. Russell is concerned with the incompatibility of both such features of causation with fundamental physical theory. It is important to note the distinctness of these two concepts. Although the failure of causal asymmetry entails the failure of causal time asymmetry (since the latter requires the discernibility of cause and effect), causal asymmetry may hold in the absence of causal time asymmetry (i.e. although causes are typically taken to precede their effects, causes could, in principle, have effects in their past). 
In the current debate, these passages by Russell are interpreted in the form of an argument called the 'Directionality Argument' (Field, 2003; Ney, 2009; Frisch, 2012). Modern advocates of the Directionality Argument draw attention to the fact that Russell observes that a paradigm case of fundamental dynamical laws of physics (the law of gravitation) is time-symmetric (Russell, 1913, pp. 15, 18, 22). Contemporary versions of the Directionality Argument cannot, of course, rely on the laws of classical mechanics as fundamental dynamical laws of physics. Today, Russellians consider other paradigmatic laws - such as the field equations of general relativity theory and the Schrödinger equation of quantum mechanics - to instantiate the feature of time symmetry. That is, the dynamical equations express time-symmetric functional relations between magnitudes. However, in ordinary and special science discourse, causation is typically taken to be time-asymmetric. Russellians contend that the time symmetry of the fundamental dynamical laws of physics contradicts the claim that there is fundamental physical time-asymmetric causation. (This claim depends on a background assumption that we make explicit below.) Russellians conclude that there are no causal relations in fundamental physics.

For the purposes of this paper, we take the Directionality Argument to run as follows:

\section{The Directionality Argument}

1. If the dynamical laws of fundamental physics are causal laws, then they are timeasymmetric (among other folk features of causation).

2. The fundamental physical theories/laws are time-symmetric.

3. Therefore, the fundamental physical theories/laws are not causal. $\quad[1 \& 2$, modus tollens]

The Directionality Argument crucially depends upon the relevance of the 'time symmetry' of physical theories to the asymmetry and time asymmetry of causation. As such, the issue with which the rest of our paper is concerned is what it means for physical theories to be 'time-symmetric' in a way that is relevantly analogous to, and comparable with, causal asymmetry and causal time asymmetry as defined above. Our goal is to assess the coherence and success of the Directionality Argument. ${ }^{2}$ We do so by clarifying the content of premises 1 and 2 and the structure of the argument. Our central focus

\footnotetext{
${ }^{2}$ However, there are challenges to the Directionality Argument that we cannot address in this paper. For example, the combination of the CPT theorem and the experimental CP-violation in neutral K-mesons (cf. Sachs, 1987, chs. 8-9) suggests that fundamental physics is not time-symmetric, contra Russell.
} 
concerns the proper interpretation of 'time symmetry' required for a plausible version of the Directionality Argument. The use of 'time-symmetric' in the current literature on the Directionality Argument is ambiguous, and as such the content and structure of the argument is unclear. We focus on two distinct senses in which physical theories can be 'time (a)symmetric' that are putatively relevant to the Directionality Argument: the timereversal invariance of physical theories; and the bidirectional nomic dependence relations of physical theories. We argue that the Directionality Argument is defendable only on the latter of these disambiguations of 'time symmetry'.

It is important to emphasise that the success of the Directionality Argument in general, and of the revised version we promote, are conditional on a further-often implicit'Russellian' background assumption. The Russellian wishes to establish that causal facts do not belong to the ontology of fundamental physics. However, this claim is not the conclusion of the Directionality Argument-which is the claim that the fundamental physical laws are not causal. The claim that causal facts are not physically fundamental follows from the conclusion of the Directionality Argument (by modus ponens) only if one accepts a further methodological principle:

Fundamental Commitment Principle. One is allowed to accept causal facts as fundamental iff causal facts are explicitly stated by the laws of fundamental physics, i.e. causal relations (characterised by 'folk' features) are explicitly referred to by the physical laws. $^{3}$

Russellians often embrace this methodological view concerning under which conditions one is allowed to accept causal facts as part of the ontology of fundamental physics (e.g. Ladyman and Ross, 2007; Loewer, 2012). We stress that the Fundamental Commitment

\footnotetext{
${ }^{3}$ This assumption is restricted to a specific part of physical theories: the dynamical laws. However, addressing the Fundamental Commitment Principle, Mathias Frisch argues that "[w]hile the dynamical equations of many of our mature theories of physics are time-reversal invariant, this does not imply that the physical theories as a whole are time symmetric" (Frisch, 2012, p. 314; emphases in original). Frisch argues that causal notions can and do play important explanatory and inferential roles in physical theories. We would like to sketch two brief responses to Frisch's view: Firstly, suppose one accepts that a physical theory can be read as causal only if its dynamical equations have specific causal features. If so, the Directionality Argument is still unsound if one reads 'time-symmetry' as time-reversal invariance (as we argue in Section 2). Secondly, suppose that Frisch is right and the Fundamental Commitment Principle is rejected. This case poses a challenge to someone who wants to endorse the Directionality Argument in the form presented in Section 3. The soundness of the argument crucially depends on the Fundamental Commitment Principle. We acknowledge that a Russellian using the Directionality Argument has to meet Frisch's challenge of providing a convincing argument for the Fundamental Commitment Principle; see Frisch $(2009 a, b, 2012)$ and Norton (2009) for a discussion.
} 
Principle imposes only an epistemological constraint concerning whether one is entitled to believe in the existence of fundamental causation, ${ }^{4}$ and not a metaphysical constraint on what sorts of world are possible. ${ }^{5}$

It is not the goal of this paper to defend the Fundamental Commitment Principle. We treat it as a background commitment of Russellians, which can, of course, be called into question and anyone opposed to it will, ultimately, not be convinced by the argument presented in this paper. However, as a referee has noted, Price and Weslake (2010, sec. 1.3) provide an argument supporting the Fundamental Commitment Principle. ${ }^{6}$ They argue that if causal facts are not explicitly described by fundamental physics and one claims that causal facts are fundamental and 'extra-scientific' (as the 'hyperrealist" does), then "the main difficulty [...] is that in putting causation beyond physics, it threatens to make it both epistemologically inaccessible and practically irrelevant." That is, the motivation for holding a methodological rule such as the Fundamental Commitment Principle is that it precludes commitment to fundamental facts that are epistemically inaccessible via our most fundamental science, physics. We acknowledge that this is one possible way to argue for the assumption, but we do not commit to it being the only argument in support of the Fundamental Commitment Principle.

The aim of this paper is to assess whether, given the Fundamental Commitment Principle, there is a convincing version of the Directionality Argument. The paper is structured as follows. Sections 2 and 3 assess the status of the Directionality Argument in light of two different readings of 'time symmetry'. In Section 2, we consider reading 'time symmetry' as the time-reversal invariance of a theory. We argue that this reading does not rule out a causal interpretation of the theory. However, the Russellian need not rely on time-reversal invariance at all in order to defend the Directionality Argument. In Section 3 , we present the alternative reading of 'time symmetry': bidirectional nomic dependence.

\footnotetext{
${ }^{4}$ Many Russellians today acknowledge the existence of causal facts as (objective) non-fundamental higherlevel facts, as referred to by the causal claims in special science and everyday contexts. One self-declared task of Russellians is to reconcile the existence of higher-level causal facts with an acausal world of fundamental physics (see Section 4 for Albert and Loewer's account).

${ }^{5} \mathrm{~A}$ referee queries whether we are committed to the rejection of the metaphysical possibility of a world with primitive causal facts in which the physics does not motivate a belief in such facts. We allow for such worlds, but hold merely that the Fundamental Commitment Principle precludes a justified belief in fundamental causation in such a world.

${ }^{6}$ Price (1996, p. 154) presents an earlier version of this argument.

${ }^{7}$ According to hyperrealism (cf. Price, 1996, p. 154; Price and Weslake, 2010, sec. 1.3), causal facts are fundamental, but not contained within the ontology of fundamental physics. This primitivist metaphysics of causation contrasts with the ontological commitment of the Russellian, as dictated by the Fundamental Commitment Principle.
} 
It is argued that if one takes the nomic dependencies captured by the equations of physical theories to represent 'ontic' dependencies in the world, then the bidirectionality of such nomic dependencies would entail that such ontic dependencies were also (temporally) bidirectional, and consequently incompatible with the asymmetry and time asymmetry of causation. The result of Section 3 is a revised and plausible version of the Directionality Argument. Section 4 considers consequences of the revised Directionality Argument for the broader debate on causation: (a) whether fundamental bidirectional ontic dependence excludes the existence of higher-level causal facts (as referred to by causal claims in everyday and special science contexts); and (b) whether the concept of bidirectional ontic dependence itself constitutes a 'scientifically updated' notion of causation, albeit a symmetric and time-symmetric one.

\section{Time Symmetry as Time-Reversal Invariance?}

The 'time symmetry' of physical theories is central to the Directionality Argument. However, it is not clear to what 'time symmetry' amounts. In the recent literature, Field (2003, p. 436), Ney (2009, p. 747) and Frisch $(2012$, p. 320) each refer to the notion of the relevant laws having the "same character" in both temporal directions as key to the Directionality Argument. However, this terminology is ambiguous; how is one to assess what constitutes sameness of 'character'? There are (at least) two importantly distinct features of physical laws/theories to which this can refer.

Firstly, the obvious formal feature of physical theories to which this relates is timereversal invariance (see, for instance, Albert [2000] and Loewer [2007; 2008] who explicitly and exclusively refer to this feature). Secondly, a physical theory can be 'time symmetric' insofar as, given some particular state of a system, the laws of the theory entail the history of the system (either deterministically or indeterministically) in both temporal directions. We call this feature 'bidirectional nomic dependence'. It is this latter feature of laws, rather than time-reversal invariance, with which Russell is apparently concerned in his discussion of the law of gravitation's equal "determination" of the past and future. Indeed, Field, Ney and Frisch each make reference to this feature of theories in addition to that of time-reversal invariance. However, given that in each case the discussions of time-reversal invariance and bidirectional nomic dependence are interspersed, we contend that these notions stand in need of further separation in the context of the Directionality Argument. In particular we wish to emphasise that the failure of a theory to be time-reversal invariant 
does not preclude it from being nomically bidirectional, and it is the nomic bidirectionality of physical theories, we argue, that is of greater relevance to the Directionality Argument. ${ }^{8}$

Our aim in sections 2 and 3 is to examine the plausibility of the Directionality Argument in light of each of these two competing interpretations of 'time symmetry', beginning in the present section with a discussion of time-reversal invariance.

\subsection{The Philosophical Interpretation of Time-Reversal Invariance and the Causal Interpretation of Theories}

On the first reading of the 'time symmetry' of fundamental physical theories, such theories are invariant under the action of time reversal.

T-SYM-1. The fundamental physical theories are 'time-symmetric' iff they are timereversal invariant.

We now argue that this reading of 'time symmetry' results in an implausible version of the Directionality Argument, because the time-reversal invariance of fundamental physical theories does not clearly preclude these theories from being interpreted causally.

As we have seen, the Directionality Argument rests on the claim that the time symmetry of the relevant physical theories is incompatible with the time asymmetry of fundamental causation. If we read 'time symmetry' as time-reversal invariance, then the argument requires an incompatibility between time-reversal invariance and causation. Call this the 'incompatibility' premise (INC-1):

INC-1. The time-reversal invariance of the fundamental physical theories is incompatible with interpreting the fundamental physical theories as causal.

We shall now demonstrate that INC-1 fails.

\footnotetext{
${ }^{8}$ For instance, Field, although explicitly discussing the bidirectional entailment of past and future states of a system by "time symmetric" laws, also defers to complications concerning 'time symmetry' by citing Sklar's (1977) critical discussion of time-reversal invariance (Field, 2003, fn. 1). Furthermore, Ney holds that "if the fundamental dynamical laws have the same character in both temporal directions, then there is no temporal asymmetry to be found in fundamental physics" (Ney, 2009, p. 748). However, if by 'sameness of character' she is referring only to nomic bidirectionality, then this is false, since a law can entail a state's past and future histories despite being time-reversal non-invariant (we elaborate on this point below), and hence her use of 'same character' appears to appeal ambiguously to both nomic bidirectionality and time-reversal invariance. See Section 3.2 for further details of the relationship between time-reversal invariance and bidirectional nomic dependence.
} 
A theory is 'time-reversal invariant' if and only if the application of a time-reversal operation to its set of solutions preserves that set of solutions. In other words, if time reversal takes each model of a theory $T$ to a model of $T$, then $T$ is time-reversal invariant. The philosophical controversy concerns to what time reversal amounts. ${ }^{9}$ On the standard 'textbook' account (cf. Sachs [1987]) time reversal acts on a history of states, $S_{i}, \ldots, S_{f}$ (where $S_{i}$ and $S_{f}$ are the initial and final states of the history), by inverting them with respect to time to $S_{f}, \ldots, S_{i}$. However, this is complicated even in such a simple case as Newtonian particle mechanics, where a state of a system is the complete specification of the position and velocity of each particle at a time. This is because in this case a time-reversal operation that merely inverts the sequence of states fails to secure the timereversal invariance of the theory, as unless the velocity of each particle is also inverted, the time-reversed sequence violates the theory. Thus, even in this simple case, a time-reversal operation on instantaneous states ${ }^{10}$ (e.g. by reversing velocities) must be introduced. Horwich (1987) and Albert (2000) each argue that a time-reversal operation intuitively ought not to operate on instantaneous states, with Albert rhetorically asking "[w]hat can it possibly mean for a single instantaneous physical situation to be happening "backward"?" (Albert, 2000, p. 18). This perceived lack of justification for time-reversing instantaneous states leads Albert to conclude that none "of the candidates for a fundamental theory that anyone has taken seriously since Newton" are time-reversal invariant (Albert, 2000, p. 14). ${ }^{11}$ For the purpose of our discussion, take 'time-reversal invariance' to be defined as follows:

Time-Reversal Invariance. A theory is time-reversal invariant iff for any history $S_{i}, \ldots$, $S_{f}$ allowed by the theory, the history $S_{f}{ }^{*}, \ldots, S_{i}{ }^{*}$ is also allowed by the theory, where $S^{*}$ is the time reverse of $S$, as determined by the time-reversal operator.

As such, time-reversal invariance is a symmetry of a theory, which can be understood in terms of how the models of the theory are related in terms of some particular time-reversal transformation.

The empirical success of a time-reversal invariant theory does not entail any particular metaphysics of the (time) (a)symmetry of causation. In order to hold that the time-reversal invariance of a theory $T$ undermines a causal interpretation of $T$ (as INC-1 states), one must

\footnotetext{
${ }^{9}$ See Albert (2000, ch. 1), Earman (2002), Malament (2004) and Arntzenius and Greaves (2009) for important recent discussions of time reversal and the time-reversal operator.

${ }^{10} \mathrm{An}$ instantaneous state is a configuration of values of the physical variables of a system at a time.

${ }^{11}$ Albert (2000) does however take such theories to display a 'curious vestige' of time-reversal invariance.
} 
hold something like the following to be true: if the time-reversal invariant theory $T$ implies that state $S_{1}$ produces (or determines) state $S_{2}$ by means of the dynamics of the theory, then-in virtue of its time-reversal invariance- $T$ also implies that it is true for the same states that $S_{2}$ produces (or determines) $S_{1}$. However, this claim relies on a specific and problematic metaphysical interpretation of time-reversal invariance.

As we state above, time reversal does not, in general, simply take a sequence of states and reverse its time order. Rather, time reversal involves a time-reversal operation on instantaneous states, such that the time-reverse of some sequence of states $S_{i}, \ldots, S_{f}$ is a sequence of distinct states $S_{f}{ }^{*}, \ldots, S_{i}{ }^{*}$, related to the first by the time-reversal operation, in reverse temporal order. To overcome this problem, one could nonetheless adopt a 'passive' interpretation of the time-reversal operation, i.e. hold that two states related by a time-reversal operation are different descriptions of the same state of affairs. However, this is itself a substantial and controversial claim that is not forced by the formal time-reversal invariance of a theory. To point to the literature on this controversy, Earman (1974, sec. 4 ) is strongly critical of such passive readings of time-reversal invariance in the work of Reichenbach (1956), Gold (1962) and Black (1962), each of whom hold that the time-reversal invariance of fundamental physics allows one to understand pairs of models related by a time-reversal transformation as alternative, equivalent descriptions of a single state of affairs. ${ }^{12}$ In particular, Earman argues that it is unjustified to understand time reversal as a passive symmetry without equally understanding other discrete symmetries such as charge conjugation and parity reversal, which are generally treated as active symmetries, as also being passive symmetries. ${ }^{13}$

We do not wish to enter into the details of this controversy, but only to note that it is sufficient to cast doubt on the legitimacy of taking the passive reading to be the 'correct' metaphysical reading of a theory's time-reversal invariance, as is required by INC-1. Quite simply, there is no overwhelming reason to hold that two states related by time reversal are the same state differently described, and in the absence of such a reason, the time-reversal invariance of a theory does not force us to hold a non-causal reading of that theory. Due to this underdetermination of the philosophical significance of a theory's time-reversal invariance, it follows that INC-1 is unsuccessful, and consequently so is the time-reversal invariance-based Directionality Argument.

\footnotetext{
${ }^{12}$ Reichenbach holds that in the case of a time-reversal invariant theory, "positive and negative time supply equivalent descriptions, and it would be meaningless to ask which of the two descriptions is true" (Reichenbach, 1956, pp. 31-32).

${ }^{13}$ See Price (2011, sec. 3.6) for a recent and illustrative discussion of this controversy.
} 
The Russellian could defend the passive reading of time reversal, but we stress that this requires him/her to engage in the further debate concerning the numerical distinctness of time-reversal-related pairs of states/models, and hence provide some further argumentation in favour of the passive reading of time-reversal symmetry. However, the Russellian need not rely on time-reversal invariance at all in order to defend the Directionality Argument: in Section 3, we argue that in any case the Russellian ought to be concerned with whether the fundamental physical theories are nomically bidirectional, since it is this, rather than time-reversal invariance, that is necessary for the success of the Directionality Argument.

\subsection{Time-Reversal Invariance and Primitive Causation}

Due to the controversy of the philosophical interpretation of time-reversal invariance, it is at least far from clear that time-reversal invariance precludes a defender of the Fundamental Commitment Principle from interpreting a theory causally. It is illustrative to consider how a time-reversal invariant theory might be read causally. A contemporary proponent of the view that time-reversal invariant theories can be understood causally is Tim Maudlin (2007), who interprets the time-reversal invariance of a theory as follows:

[I]f $\left[S_{i}, \ldots, S_{f}\right]$ is physically allowable as time runs from $\left[S_{i}\right.$ to $\left.S_{f}\right]$ in some direction, then $\left[S_{f}^{*}, \ldots, S_{i}^{*}\right]$ is also allowed $[\ldots]$, where time runs from $\left[S_{f}^{*}, \ldots, S_{i}^{*}\right]$ in the same direction as it runs from $\left[S_{i}\right.$ to $\left.S_{f}\right]$. (Maudlin, 2007, p. 119, emphasis in original.)

Maudlin's point is that a theory can satisfy the criterion for time-reversal invariance without this entailing that, according to the theory, whatever can happen 'forwards' in time can happen 'backwards' in time. Rather, the time-reversal invariance of a theory entails a weaker conclusion: if some sequence of states is allowed by the theory, then a second sequence of states is also allowed by the theory, where the second sequence consists of the time reverse of each state (determined by the time-reversal operator) in reverse temporal order to that of the original sequence. As Maudlin notes, this can be satisfied even if such sequences only 'occur' in one temporal direction.

Maudlin argues that the time-reversal invariance of a theory in itself does not entail any particular metaphysical view with respect to the directionality of time. Maudlin, for instance, holds that the dynamical laws of fundamental physics ('fundamental laws of 
temporal evolution') describe the 'passage' of time, which he understands as a metaphysically primitive time-asymmetric 'production' relation holding between states of the entire universe such that earlier states 'produce' later states (cf. Maudlin, 2007, pp. 14-21, ch. 4). Maudlin argues that the existence of primitive production relations is consistent with the fundamental physical theories being invariant with respect to time reversal. This point is quite general and not restricted to Maudlin's particular view: if one posits metaphysically fundamental causal relations (such that it is metaphysically primitive that 'earlier' states cause 'later' ones), then one can understand a time-reversal invariant theory as describing metaphysically primitive causal facts.

However, the problem with this type of view in the present context is that, in order to satisfy the Russellian, such a theory of causation must not violate the Fundamental Commitment Principle, and hence must be motivated by the physics. In the context of the Directionality Argument, Russellians are concerned only with whether the physics motivates a belief in causal relations, and not whether the physics is merely consistent with a primitive, non-physical causal relation. In contrast, Maudlin's own metaphysics of unidirectional 'production' has a primarily extra-physical motivation, insofar as he takes the belief in a fundamental passage of time to be justified by the fact that "the world is given to us as changing, and time as passing, [...] and that all the philosophizing in the world will not convince us that [this is a] mere illusion" (Maudlin, 2007, p. 135). ${ }^{14}$

To avoid a violation of the Fundamental Commitment Principle, the defender of fundamental causation, we suggest, could rely on a further property of fundamental physical theories. That is, an advocate of fundamental causation might argue that, given some state of a system, the relevant physical theory nomically entails only states in one temporal direction from it. If the fundamental physical theories had this property of nomic unidirectionality, then the advocate of fundamental causation could legitimately infer that the laws had the requisite asymmetry and time-asymmetry to warrant a causal interpretation without violating the Fundamental Commitment Principle. Conversely, if the laws were nomically bidirectional, then they would not warrant a causal interpretation. In Section 3,

\footnotetext{
${ }^{14}$ Maudlin does however argue (Maudlin, 2007, pp. 131-135) that positing unidirectional production has explanatory value in the case of the statistical mechanical explanation of the entropy gradient. In order to explain the entropy gradient, Maudlin relies on the notion of typicality in the case of microstates. He claims that primitive production relations explain away part of the atypicality of microstates that lead to lower entropy towards the past. As such, it could be argued that Maudlinian 'production' is motivated by the physics, because production features in the best explanation of atypical microstates, and hence positing primitive production satisfies the Fundamental Commitment Principle. See Loewer (2012) and Farr (2013, ch. 7, sec. 7.6) for analyses and criticisms of Maudlin's argument.
} 
we argue that a bidirectional nomic dependence-based Directionality Argument is more fruitful than a time-reversal invariance-based Directionality Argument.

We are now in a position to evaluate the time-reversal invariance-based Directionality Argument. Firstly, the time-reversal invariance-based Directionality Argument is unsuccessful because INC-1 fails. In order to support the time-reversal invariance-based Directionality Argument, the Russellian must provide independent justification for the passive interpretation of time-reversal. Secondly, in order to hold that that a time-reversal invariant theory is causal, without violating the Fundamental Commitment Principle, one requires a further property of physical theories, namely that they be nomically unidirectional. In Section 3, we consider the status of the Directionality Argument in light of this alternative feature of physical theories by reading 'time symmetry' as 'bidirectional nomic dependence'.

\section{Time Symmetry as Bidirectional Nomic Dependence- Russell's Legacy}

We shall now introduce a more promising, alternative way of understanding the time symmetry of physical laws/theories: bidirectional nomic dependence. As the current literature has not explicitly treated bidirectional nomic dependence in isolation from timereversal invariance, we believe that understanding time symmetry as bidirectional nomic dependence provides a novel perspective on the Directionality Argument. A physical theory has the property of bidirectional nomic dependence iff its dynamical laws are such that, given some state $S$ of a system, the laws determine the history of the system (or multiple possible histories of the system in case of indeterministic laws) in both temporal directions from $S$. The contention of this section is that this feature of physical theories, unlike time-reversal invariance, is of direct relevance to the Directionality Argument, and allows for a plausible version of the Directionality Argument. (We provide a substantive definition of bidirectional nomic dependence in Section 3.1.) The crucial dialectical point of Section 3 is that we read 'time symmetry', as it figures in the Directionality Argument, as follows:

T-SYM-2. The equations of the fundamental physical theories are time-symmetric iff they express only bidirectional nomic dependence relations between the states of a physical system at different times. 
An immediate advantage of this interpretation of time symmetry is that it is this, rather than time-reversal invariance, with which Russell is more obviously concerned. Consider the quotations of Russell in Section 1. Russell explicitly speaks of the 'calculability' of the past and future in terms of the differential equations of physical theories and the present state, and of the consequent 'logical determination' of the past and future by these equations.

Our aim in this section is to assess the following incompatibility claim (INC-2):

INC-2. The bidirectionality of the nomic dependence relations expressed by the equations of fundamental physical theories is incompatible with the causal interpretation of the laws of these theories.

We demonstrate below that INC-2, unlike INC-1, is plausible and that, given this, interpreting 'time symmetry' as bidirectional nomic dependence results in a promising revised version of the Directionality Argument.

\subsection{What is Bidirectional Nomic Dependence?}

Bidirectional nomic dependence is expressed most clearly by deterministic theories. If a theory is deterministic, then, for a system governed by the theory, its state at any one time is sufficient for the theory to determine its entire history, and thus to give the state at any other ('past' or 'future') time. A natural way to understand nomic dependence is nomic entailment (in the deterministic ${ }^{15}$ case). That is, a state $A$ nomically depends on a state $B$ iff $B$ and the laws entail the occurrence of $A$. For example, the wavefunction $\Psi_{1}$ of a system at $t_{1}$ is nomically dependent upon the wavefunction $\Psi_{0}$ of the system at an earlier time $t_{0}$ iff $\Psi_{0}$ and the (time-dependent) Schrödinger equation entail $\Psi_{1}$. If the dependence is bidirectional, then the laws plus $A$ also entail $B$-which is to say that, if the dependence expressed by the Schrödinger equation is bidirectional, then $\Psi_{1}$ and the Schrödinger equation also entail $\Psi_{0}{ }^{16}$ In order to avoid misunderstandings, we stress that

\footnotetext{
${ }^{15}$ In the case of indeterministic laws, nomic entailment consists in the entailment of a probability that a state $A$ occurs by the laws plus a state $B$. For a discussion of bidirectional nomic dependence in indeterministic theories see Farr (2012, sec. 4.2-4.4).

${ }^{16}$ Nomic dependence need not be spelled out as nomic entailment. As a referee has pointed out, dependence can be alternatively understood as counterfactual dependence. Given this reading, two states $A$ and $B$ are bidirectionally dependent iff (i) $A$ counterfactually depends on $B$, and (ii) $B$ counterfactually depends on $A$. Although we are sympathetic to this approach, we would like to address the question as to whether there is a convincing account of bidirectional counterfactual dependence in a separate paper. One important reason to adopt this strategy is that bidirectional counterfactual dependence implies the controversial claim
} 
in what follows we understand unidirectional as well as bidirectional nomic dependence in terms of nomic entailment.

Russell refers to bidirectional dependence by using the concept of symmetric 'determination' relations between states of a physical system at different times. ${ }^{17}$ Russell illustrates 'determination' by identifying the feature of bidirectional dependence in the case of the law of gravitation, and takes this to show that, according to the law, "the future 'determines' the past in exactly the same sense in which the past 'determines' the future", where "a certain number of variables 'determine' another variable if that variable is a function of them" (Russell, 1913, p. 15).

\subsection{Disentangling Bidirectional Nomic Dependence and Time-Reversal Invariance}

Since we contend that disentangling the concepts of time-reversal invariance and bidirectional nomic dependence serves to clarify the Directionality Argument, it is important to establish how these two features of physical theories can be properly distinguished. The key point we wish to stress is that, although it may be the case that a nomically unidirectional theory must also be time-reversal non-invariant, the converse is not true: the time-reversal non-invariance of a theory does not entail that the theory is nomically unidirectional. As such, the question of whether a theory is nomically unidirectional or bidirectional is of more basic relevance to the Russellian than is the question of whether the theory is time-reversal invariant.

The following illustrates that time-reversal non-invariance does not entail unidirectional nomic dependence. Take a theory $T$ to be composed of a single law $L$, and take $L$ to hold that some physical magnitude $M$ increases by one unit per second. $T$ is, intuitively, time-reversal non-invariant- the natural ${ }^{18}$ time reverse of a sequence of states according to which the relevant magnitude increases in accord with $L$ is a sequence of states according to which the relevant magnitude decreases, in violation of $L$. However, this does not entail that $T$ lacks the feature of bidirectional nomic dependence. If a system has an $M$-value of 2

that earlier states depend on later states (involving so-called backtracking counterfactuals). Due to space constraints we cannot do justice to a counterfactual reading of dependence relations.

${ }^{17}$ Our argument does not depend on Russell's definition of determinism; see Earman (1986, pp. 10-12) for a critical discussion.

${ }^{18}$ One can nonetheless manufacture a time-reversal operation for the theory such that the value of $M$ is, for example, is taken to $-n$, such as to ensure that the time reverse of every model of $T$ is a model of $T$. We take it to be clear as to why this is to be considered not a natural time-reversal operation for $T$. 
at time $t_{0}$, then the theory entails (e.g.) that the value of $M$ at $t_{1}$ is 3 , and it entails (e.g.) that the value of $M$ at $t_{-1}$ is 1 . Thus even though $T$ is intuitively time-reversal non-invariant, it satisfies bidirectional nomic dependence.

\subsection{A Realist Interpretation of Nomic Dependence}

We have introduced bidirectional dependence as a nomic concept. However, we wish to avoid understanding this merely as a property of theories (either as sets of models or of sentences), or as an epistemic notion concerning a theory's ability to calculate earlier and later states in equal measure. Rather, in order to relate nomic dependence to causation, we propose a realist reading of the nomic dependencies expressed by physical theories. According to this realist reading, nomic dependencies correspond to ontic dependencies holding between token states of the actual world, and as such, bidirectional nomic dependence is directly comparable to the features of causation with which the Directionality Argument is concerned.

As introduced above, a state $A$ of a system nomically depends upon a different state $B$ of the same system iff $A$ is entailed by $B$ and some dynamical law. If one wishes to make a claim about the metaphysics of laws and causation, then one needs a metaphysically more robust-a realist-interpretation of nomic entailment. If we are to look to physical theories to understand whether causal relations are part of the ontology of fundamental physics (and whether the laws of the theory in question can be interpreted causally), it is necessary that we take features of the physical theories to correspond to features of the world. It is our contention that in order to construct a physical theory of causation, one must take the nomic dependencies of physical theories to represent ontic dependence relations between token world states. If theories express bidirectional dependence relations between physical states, then this corresponds to the mind-independent existence of dependencies of (actual) world states upon earlier and later world states. For the sake of convenience, we use the label 'ontic dependence' for dependence between states. ${ }^{19}$

If we take the nomic dependencies of the fundamental physical theories as representing ontic dependencies in the world, then INC-2 entails the following two claims: (1) on the assumption that the nomic dependence relations of fundamental physical theories

\footnotetext{
${ }^{19}$ Two disclaimers: (1) the realist interpretation is neutral with respect to the question of whether the dependence relation itself is fundamental—a question to which Humeans and anti-Humeans provide different answers; (2) ontic dependence as used here is not to be confused with the notion of metaphysical dependence, which is the subject of the metaphysics literature on supervenience, constitution, and grounding.
} 
are bidirectional, the corresponding ontic dependence relations represented by them are also bidirectional and, hence, not causal; (2) if the nomic dependence is unidirectional, then the ontic dependence relations must be unidirectional and, hence, support a causal interpretation. That is, if causation were a physically fundamental relation expressed by the laws of fundamental physical theories, then the laws would represent dependence relations of world states at some time $t$ upon earlier ${ }^{20}$ world states only. ${ }^{21}$

Recall the two characteristic folk features of causation that Russell takes to be incompatible with the symmetric and time-symmetric nature of the kind of dependence expressed by the laws of fundamental physical theories: (a) the cause-effect relation is asymmetric; (b) the cause-effect relation is time-asymmetric. If we believe that the dynamical laws are the right place to look for causes (Fundamental Commitment Principle) and if the laws commit us to bidirectional (ontic) dependence relations between token world states, then the laws fail to satisfy each of causal asymmetry and causal time asymmetry.

Firstly, we can see that causal time asymmetry is inconsistent with bidirectional dependence. Given that nomically bidirectional equations describe the evolution in both temporal directions from a state, the corresponding ontic dependence relations are such that a world state is dependent upon other world states in both temporal directions. Thus, it is not the case that all such dependence relations are such that states depend only on earlier $^{22}$ states. It follows that bidirectional dependence relations do not have the feature of causal time asymmetry.

Secondly, and more significantly, bidirectional dependence violates causal asymmetry. If a system is governed by a theory with the feature of bidirectional nomic dependence, then it follows that each state of the system is nomically dependent upon each other state of the system. On the corresponding ontic interpretation, each world state is dependent upon each other world state, entailing that the ontic dependence is symmetric-for any pair of states, $S_{1}$ and $S_{2}, S_{1}$ ontically depends on $S_{2}$ iff $S_{2}$ ontically depends on $S_{1}$. It follows that such dependence relations do not have the feature of causal asymmetry. ${ }^{23}$

Summing up the reasoning of this section, we are now in a position to present a revised version of the Directionality Argument in terms of bidirectional dependence:

\footnotetext{
${ }^{20}$ It is tenable for a theory to be nomically unidirectional insofar as it holds that states depend only on later rather than earlier states. We ignore this detail since we take the opponent to the Directionality Argument, in general, to hold that causes are earlier than, rather than later than, their effects.

${ }^{21}$ See footnote 3 for a disclaimer.

${ }^{22}$ Or later-recall footnote 20.

${ }^{23}$ Moreover, recall that these symmetric and time-symmetric features of bidirectional dependence are compatible with the relevant theory being time-reversal non-invariant.
} 


\section{The Revised Directionality Argument}

1. If the dynamical theories/laws of fundamental physics are causal, then they express unidirectional nomic dependence relations.

2. The fundamental physical theories/laws express only bidirectional nomic dependence relations.

[Assumption] $^{24}$

3. Therefore, the fundamental physical theories/laws are not causal.

$[1 \& 2$, modus tollens]

\subsection{Defending the Revised Directionality Argument}

We have argued that the bidirectional nomic dependencies of a physical theory can plausibly be taken to be incompatible with causally asymmetric and time-asymmetric dependence relations. Unlike time-reversal invariance, bidirectional nomic dependence does motivate a particular non-causal interpretation of a physical theory (in particular, of the dynamical laws). We have argued that the realist reading of the bidirectional nomic dependence of physical theories involves a commitment to the existence of bidirectional ontic dependence relations between world states. Bidirectional ontic dependence relations lack the features of both causal asymmetry and causal time-asymmetry. We conclude this section by defending the Revised Directionality Argument against two objections. ${ }^{25}$

Firstly, in response to the Revised Directionality Argument, one might object that it is always possible to imagine the following scenario: there is a possible world which is described by laws expressing nomic bidirectional dependence and it is the case that primitive time-asymmetric causal facts obtain in this world. This requires one to think about causation in a different way than we have done up to this point in the present section. The causal facts are metaphysically primitive in the sense that they cannot be reduced to (or supervene on) a dependence relation of the sort we have been discussing (in sections 3.1-3.2). An example of what primitive causal facts are might be Maudlin's

\footnotetext{
${ }^{24}$ In order to assess the nomic dependence version of the Directionality Argument, we suppose, for sake of argument, that the equations of the fundamental physical theories express bidirectional nomic dependence relations. The fact that the dynamical equations of physical theories are routinely used to predict and retrodict suggests that such theories are as a matter of fact bidirectional, and it is this to which Russell makes reference in the case of gravitation. However, whether this is indeed the case is outside of the scope of this paper. We take this to be an open issue, with collapse formulations of quantum mechanics perhaps the most prominent candidates for a nomically unidirectional fundamental physical theory.

${ }^{25}$ Both objections were raised by an anonymous referee.
} 
production relations (see Section 2) and other accounts of primitive causal production (cf. Price and Weslake [2010] for a brief overview).

To avoid misunderstandings, we do not claim that understanding causal facts as primitive is unconditionally untenable. However, if the fundamental physics is nomically bidirectional, then introducing such primitive causal facts violates the Fundamental Commitment Principle; it is a form of hyperrealism (see sections 1 and 2.2). Moreover, judging whether the fundamental laws can be interpreted causally by understanding causation in terms of (unidirectional) nomic dependence has an epistemic advantage over hyperrealism. One can, in principle, read off the theory whether or not the laws are causal. If the laws express unidirectional dependence (i.e. later states depend on earlier states and not vice versa), then causal relations are part of the ontology of fundamental physics. If the laws express only bidirectional dependence, then causal relations are not part of the ontology of fundamental physics. In contrast, we claim (in accordance with Price and Weslake's [2010] argument [see Section 1]) that hyperrealists face an epistemological problem since their primitive causal facts lie beyond the grasp of physics. ${ }^{26}$

Secondly, one might object that a bidirectional law might still express causal dependencies. For instance, one might claim that a cause could be nomologically sufficient for its effect and also the effect nomologically sufficient for the cause. In other words, the fact that a dependence relation is bidirectional does entail that the dependence is non-causal. If we understand this second objection correctly, then it differs from the first objection (that relies on positing primitive causal facts), because in the case of the second objection causation is understood in terms of dependence. In response, we contend that if the laws allow us to determine sufficient conditions for later as well as earlier events (this is one type of bidirectional nomic dependence), then we lack reason to speak of causal laws at all. We support this claim with two points. (a) In the debate on causation, it is generally recognised as a problem if a particular theory of causation cannot capture the (time) asymmetric character of the (dependence) relation between cause and effect. One of the

\footnotetext{
${ }^{26}$ Aside from violating the Fundamental Commitment Principle, the hyperrealist view does not seem to be viable for all cases of nomically bidirectional theories. For example, the laws of physics could be such that some particular state $X$ of a system is only nomically entailed by the laws conditional upon an earlier state and a later state. The two-state vector formalism (TSVF) of quantum mechanics' account of the weak value of a system has precisely this form. The hyperrealist must either reject the reality of weak values or adopt an alternative understanding of them to that of TSVF. Neither option is untenable, but each places an a priori constraint on physical explanation that is both physically unmotivated and inconsistent with a currently active research programme. We take the job of the philosopher of physics to be to allow current physics to dictate which philosophical positions are tenable, and not vice versa. See Aharonov et al. (2010) for a summary of recent work on weak values.
} 
major goals and one of the hard problems in this debate is to establish that the dependence relation in question is (time) asymmetric. For instance, to refer to only a few voices in a large literature, Lewis (1979) attempts to establish unidirectional dependence in the case of counterfactual theories, Mackie (1974, ch. 7) in the case of the regularity theory, and Dowe (2000, ch. 8) for the conserved quantity theory. (b) In the causal modelling literature, it is an explicit assumption that structural equations express unidirectional dependence relations between variables, and it is, ultimately, this feature of unidirectional dependence that warrants a causal interpretation of these equations (Pearl, 2000, p. 27). Interventionist theories of causation openly rely on this feature of structural equations (Woodward, 2007, pp. 318-327). ${ }^{27}$

\section{Conclusion and Outlook}

We have argued that the soundness of the Directionality Argument crucially depends on the disambiguation of the term 'time-symmetric' figuring in both premises of the argument. We have considered two alternative readings of 'time-symmetric'. In Section 2, we have argued that time-reversal invariance is in general not the adaquate interpretation of 'time symmetry'. If we interpret 'time symmetry' as time-reversal invariance, then the defender of the Directionality Argument must provide independent grounds for the objective preference of the passive interpretation of time-reversal invariance. In the absence of such grounds, we take this version of the Directionality Argument to fail. Furthermore, we have argued that the time-reversal invariance of the fundamental physical theories is not even necessary for a successful version of the Directionality Argument. In Section 3, we have argued that the reading of 'time symmetry' necessary for the success of the Directionality Argument is bidirectional nomic dependence. We have furthermore suggested a realist interpretation of bidirectional nomic dependence. If the fundamental dynamical laws have the feature of bidirectional dependence, then they lack the features of causal time asymmetry and causal asymmetry. Bidirectional nomic dependence is incompatible with a causal interpretation of the laws (that is, more precisely, with a causal interpretation that does not violate the Fundamental Commitment Principle). Furthermore, we have argued that the bidirectional dependence interpretation of 'time symmetry' is a more accurate reconstruction of Russell's original argument than is the time-reversal invariance

\footnotetext{
${ }^{27}$ However, if one insists that causation can be understood in terms of dependence and that it is also possible that this dependence is bidirectional, then one faces a similar problem to Ney (2009), which we address in Section 4.
} 
interpretation.

We conclude the paper with a brief discussion of the impact of the Revised Directionality Argument on the current debate on causation. We discuss two examples.

Firstly, one might wonder whether the Revised Directionality Argument eliminates causation entirely. This concern is not unmotivated, since Russell's view is often described as eliminating causation. For instance, someone who works on causal explanation in the life sciences might worry that the Revised Directionality Argument shows that there is no time-asymmetric causation and hence threatens their own research. Fortunately, we think this worry is misplaced. The conclusion of the Directionality Argument is that the fundamental laws are not causal, and - given the Fundamental Commitment Principleit follows that causal relations do not belong to the ontology of fundamental physics. Call this claim 'the Russellian claim'. However, the Russellian claim does not rule out the possibility that causal facts are non-fundamental higher-level facts. Call the claim that causal facts are higher-level facts 'the higher-level claim'. The higher-level claim is supported by: (a) the successful use of causal concepts in the special sciences; and (b) by our everyday experience as agents when causally interacting with the world around us.

Many neo-Russellians wish to explain how both the Russellian claim and the higherlevel claim can be true in the actual world. David Albert and Barry Loewer advocate one prominent strategy to meet this challenge (Albert, 2000; Loewer, 2008).$^{28}$ In virtue of being Russellians, Albert and Loewer believe that the fundamental dynamical laws are non-causal (Loewer, 2008, p. 151). However, Albert and Loewer observe, these dynamical laws are not sufficient (although necessary) to explain striking phenomena that happen on the macroscopic scale. Their central idea is that, in order to explain the existence of higher-level causal facts, one needs other assumptions in addition to the time-symmetric dynamical laws. Albert and Loewer identify two crucial additional assumptions. The first assumption is the past hypothesis $(\mathrm{PH})$, which states that the initial macrostate of the universe was low-entropy. The second assumption required for an explanation of timeasymmetric higher-level causal facts is the statistical postulate PROB, which states that there is a uniform probability distribution over the physically possible initial microstates of the universe compatible with $\mathrm{PH}$ (i.e. the possible realisers of the initial macrostate referred to in $\mathrm{PH}$ ). According to Albert and Loewer, the conjunction of the time-symmetric dynamical laws, $\mathrm{PH}$, and $\mathrm{PROB}$ provides a statistical explanation of time-asymmetric

\footnotetext{
${ }^{28}$ Alternative strategies are advocated by, for instance, Hitchcock (2007), Ladyman and Ross (2007, ch. 5), Ross and Spurrett (2007), Woodward (2007), Ladyman (2008), and Reutlinger (2013, ch. 6).
} 
macro-facts (such as causal and entropic higher-level facts).

The main point we would like to stress is that it is consistent with the Revised Directionality Argument and the characterisation of time symmetry as bidirectional nomic dependence (Section 3) to say that causal facts are higher-level facts. Moreover, a proponent of the Directionality Argument can adopt Albert and Loewer's strategy in order to explain why certain higher-level causal facts obtain. Someone who is interested in causal explanations in the life sciences can refer to these higher-level causal facts.

Secondly, the further impact of the Revised Directionality Argument concerns a metaphilosophical issue. Consider the following question: what exactly do we learn from the Revised Directionality Argument? Russellians are inclined to reply that we learn that causation as characterised by the folk notion (which includes the feature of time asymmetry) is not fundamental-given the Fundamental Commitment Principle. However, another reaction is possible, which is presented by Alyssa Ney (2009). Ney holds that the Directionality Argument shows that we have to revise our folk concept of causation and replace it with a scientific concept of causation. In the particular case of the Directionality Argument, Ney holds that we ought to revise the feature of time asymmetry of our folk concept of causation, because the fundamental dynamical laws are time-symmetric. Ney defends the view that a scientifically updated concept of causation holds causation to be time-symmetric (cf. Ney, 2009, p. 760).

What should one make of this dispute between Russellians and Ney? This looks like a merely verbal dispute. The parties in the dispute are not really disagreeing about anything. Both parties agree that (a) the dynamical laws are complete and time-symmetric. Furthermore they agree that (b) the folk feature of causal time asymmetry cannot be attributed to the dynamical laws. (c) Both parties agree that the folk concept of causation and the concept of bidirectional ontic dependence (as expressed by the dynamical law statements) are different concepts. The point of disagreement concerns whether bidirectional ontic dependence deserves the name 'causation'. It seems to us that the answer to this question can only be some kind of conventional agreement on how to use the word 'cause'. We suggest that one should conventionally agree on the Russellian use of the word 'causation', because Ney's proposal to call time-symmetric dependence 'scientifically updated causation ${ }^{29}$ involves two risks.

(A) Ney's strategy provokes misunderstandings. In the current debate, most people care about: (1) whether the folk concept of causation plays a role in physical theories

${ }^{29}$ Cf. Ney (2009, sec. 5). 
and physical reasoning; and (2) whether and how causal facts-as picked out by the folk notion - can be explained by (or, speaking in a more metaphysical mode, be grounded in) non-causal particular and nomic physical facts. We think that using the same word 'cause' for another concept (bidirectional ontic dependence) is likely to lead to a situation in which philosophers talk past each other. In order to avoid confusion, we advocate restricting the word 'cause' to the folk (and special science) notion of causation, while using the concept of bidirectional ontic dependence when we refer to the kind of nomic dependence expressed by the dynamical laws of fundamental physics.

(B) It is by no means clear what difference it makes to use the label 'scientific causation' for bidirectional ontic dependence if one wishes to talk about causation in (e.g.) economics or biology. It seems that causation in these disciplines is associated with features such as causal asymmetry, time asymmetry, locality etc. If this is the case, then we have (at least) two concepts of causation: time-symmetric physical causation and time-asymmetric special science causation. However, even if we accept the revised concept of time-symmetric causation, it does not resolve, for instance, the problem with which Albert and Loewer (see above) are concerned: how can we explain that physical dependence relations are time-symmetric and special science dependence relations are time-asymmetric? Labelling time-symmetric dependence as 'causal' does not resolve the problem.

\section{Acknowledgements}

We would like to thank Andreas Hüttemann and James Ladyman, the participants of Andreas Hüttemann's colloquium in Cologne, and the members of our audiences at the conference Causality and Explanation in the Sciences in Ghent, and in Munich and Vancouver for their comments on earlier versions of the paper. We are also grateful for the comments we received from two anonymous referees for Erkenntnis. Special thanks to Bert Leuridan and Erik Weber for their editorial work. Matt's research is supported by the John Templeton Foundation grant: New Agendas for the Study of Time: Connecting the Disciplines, at the Centre for Time, University of Sydney. Alex's research is funded by the DFG Research Group Causation and Explanation (University of Cologne), and the Center for Philosophy of Science (University of Pittsburgh). 


\section{References}

Aharonov, Y., S. Popescu, and J. Tollaksen (2010). A time-symmetric formulation of quantum mechanics. Physics Today 63, 27-33.

Albert, D. Z. (2000). Time and Chance. Massachusetts: Harvard University Press.

Arntzenius, F. and H. Greaves (2009). Time reversal in classical electromagnetism. British Journal for the Philosophy of Science 60(3), 557.

Black, M. (1962). Models and Metaphors: Studies in Language and Philosophy. Ithaca: Cornell University Press.

Dowe, P. (2000). Physical Causation. Cambridge: Cambridge University Press.

Earman, J. (1974). An attempt to add a little direction to "the problem of the direction of time". Philosophy of Science 41(1), 15-47.

Earman, J. (1986). A Primer on Determinism. Boston: Dordrecht.

Earman, J. (2002). What time reversal invariance is and why it matters. International Studies in the Philosophy of Science 16(3), 245-264.

Farr, M. (2012). On A- and B-theoretic elements of branching spacetimes. Synthese 188(1), 85-116. doi: 10.1007/s11229-011-0046-y.

Farr, M. (2013). Towards a C Theory of Time: An Appraisal of the Physics and Metaphysics of Time Direction. Ph. D. thesis, University of Bristol, Bristol.

Field, H. (2003). Causation in a physical world. In M. Loux and D. Zimmerman (Eds.), Oxford Handbook of Metaphysics, pp. 435-60. Oxford: Oxford University Press.

Frisch, M. (2009a). 'The most sacred tenet'? Causal reasoning in physics. The British Journal for the Philosophy of Science 60(3), 459-474.

Frisch, M. (2009b). Causality and dispersion: A reply to John Norton. The British Journal for the Philosophy of Science 60(3), 487-495.

Frisch, M. (2012). No place for causes? Causal skepticism in physics. European Journal for Philosophy of Science 2(3), 313-336. 10.1007/s13194-011-0044-4. 
Gold, T. (1962). The arrow of time. American Journal of Physics 30(6), 403-410.

Hitchcock, C. (2007). What Russell got right. In H. Price and R. Corry (Eds.), Causation, Physics and the Constitution of Reality: Russell's Republic Revisited, pp. 45-65. Oxford: Clarendon Press.

Horwich, P. (1987). Asymmetries in Time. Massachusetts: MIT Press.

Ladyman, J. (2008). Structural realism and the relationship between the special sciences and physics. Philosophy of Science 75, 744-755.

Ladyman, J. and D. Ross (2007). Every Thing Must Go: Metaphysics Naturalized. Oxford: Oxford University Press.

Lewis, D. (1979). Counterfactual dependence and time's arrow. Noûs 13(4), 455-476.

Loewer, B. (2007). Counterfactuals and the Second Law. In H. Price and R. Corry (Eds.), Causation, Physics and the Constitution of Reality: Russell's Republic Revisited. Oxford: Oxford University Press.

Loewer, B. (2008). Why there is anything except physics. In J. Hohwy and J. Kallestrup (Eds.), Being Reduced. New Essays on Reduction, Explanation, and Causation, pp. 149-163. Oxford University Press.

Loewer, B. (2012). Two accounts of laws and time. Philosophical Studies 160, 115-137.

Mackie, J. L. (1974). The Cement of the Universe: A Study of Causation. Oxford: Oxford University Press.

Malament, D. (2004). On the time reversal invariance of classical electromagnetic theory. Studies In History and Philosophy of Science Part B: Studies In History and Philosophy of Modern Physics 35(2), 295-315.

Maudlin, T. (2007). The Metaphysics Within Physics. Oxford: Oxford University Press.

Ney, A. (2009). Physical causation and difference-making. British Journal for the Philosophy of Science 60(4), 737.

Norton, J. D. (2007). Causation as folk science. In H. Price and R. Corry (Eds.), Causation, Physics and the Constitution of Reality: Russell's Republic Revisited, Chapter 2, pp. 11-44. Oxford: Oxford University Press. 
Norton, J. D. (2009). Is there an independent principle of causality in physics? The British Journal for the Philosophy of Science 60(3), 475-486.

Pearl, J. (2000). Causality: Models, Reasoning and Inference. Cambridge: Cambridge University Press.

Price, H. (1996). Time's Arrow and Archimedes' Point: New directions for the physics of time. Oxford: Oxford University Press.

Price, H. (2011). The flow of time. In C. Callender (Ed.), The Oxford Handbook of Philosophy of Time, Chapter 9. Oxford: Oxford University Press.

Price, H. and B. Weslake (2010). The time-asymmetry of causation. In H. Beebee, C. Hitchcock, and P. Menzies (Eds.), The Oxford Handbook of Causation, pp. 414-443. Oxford: Oxford University Press.

Reichenbach, H. (1956). The Direction of Time. Berkeley: University of California Press.

Reutlinger, A. (2013). A Theory of Causation in the Social and Biological Sciences. London: Palgrave Macmillan.

Ross, D. and D. Spurrett (2007). Notions of cause: Russell's thesis revisited. British Journal for the Philosophy of Science 58(1), 45-76.

Russell, B. (1912-1913). On the notion of cause. Proceedings of the Aristotelian Society 13, pp. 1-26.

Sachs, R. G. (1987). The Physics of Time Reversal. Chicago: University of Chicago Press.

Sklar, L. (1977). Space, Time and Spacetime. Berkeley: University of California Press.

Woodward, J. (2007). Causation with a human face. In H. Price and R. Corry (Eds.), Causation, Physics and the Constitution of Reality: Russell's Republic Revisited, pp. 66-105. Oxford: Clarendon Press. 